\title{
Dynamical Critical Phenomena in Driven-Dissipative Systems
}

\author{
L. M. Sieberer ${ }^{1,2}$, S. D. Huber ${ }^{3,4}$, E. Altman ${ }^{4,5}$, and S. Diehl ${ }^{1,2}$ \\ ${ }^{1}$ Institute for Theoretical Physics, University of Innsbruck, A-6020 Innsbruck, Austria \\ ${ }^{2}$ Institute for Quantum Optics and Quantum Information of the Austrian Academy of Sciences, A-6020 Innsbruck, Austria \\ ${ }^{3}$ Theoretische Physik, Wolfgang-Pauli-Strasse 27, ETH Zurich, CH-8093 Zurich, Switzerland \\ ${ }^{4}$ Department of Condensed Matter Physics, Weizmann Institute of Science, Rehovot 76100, Israel \\ ${ }^{5}$ Department of Physics, University of California, Berkeley, CA 94720, USA
}

\begin{abstract}
We explore the nature of the Bose condensation transition in driven open quantum systems, such as excitonpolariton condensates. Using a functional renormalization group approach formulated in the Keldysh framework, we characterize the dynamical critical behavior that governs decoherence and an effective thermalization of the low frequency dynamics. We identify a critical exponent special to the driven system, showing that it defines a new dynamical universality class. Hence critical points in driven systems lie beyond the standard classification of equilibrium dynamical phase transitions. We show how the new critical exponent can be probed in experiments with driven cold atomic systems and exciton-polariton condensates.
\end{abstract}

Recent years have seen major advances in the exploration of many-body systems in which matter is strongly coupled to light [1]. Such systems include for example polariton condensates [2], superconducting circuits coupled to microwave resonators [3, 4], cavity quantum electrodynamics [5] as well as ultracold atoms coupled to high finesse optical cavities [6]. As in traditional quantum optics settings, these experiments are subject to losses, which may be compensated by continuous drive, yet they retain the many-body character of condensed matter. This combination of ingredients from atomic physics and quantum optics in a many-body context defines a qualitatively new class of quantum matter far from thermal equilibrium. An intriguing question from the theoretical perspective is what new universal behavior can emerge under such conditions.

A case in point are exciton-polariton condensates. Polaritons are short lived optical excitations in semiconductor quantum wells. Continuous pumping is required to maintain their population in steady state. But in spite of the non-equilibrium conditions, experiments have demonstrated Bose condensation [2] and, more recently, have even observed the establishment of a critical phase with power-law correlations in a two dimensional system below a presumed Kosterlitz-Thouless phase transition [7]. At a fundamental level however there is no understanding of the condensation transition in the presence of loss and external drive, and more generally of continuous phase transitions under such conditions.

In this letter we develop a theory of dynamical critical phenomena in driven-dissipative systems in three dimensions. Motivated by the experiments described above we focus on the case of Bose condensation with the following key results. (i) Low-frequency thermalization - The microscopic dynamics of a driven system is incompatible with an equilibrium-like Gibbs distribution at steady state. Nevertheless a scale independent effective temperature emerges at low frequencies in the universal regime near the critical point, and all correlations in this regime obey a classical fluctuation-dissipation relation (FDR). Such a phenomenon of low frequency effective equilibrium has been identified previously in different contexts [8-
13]. (ii) Universal low-frequency decoherence - In spite of the effective thermalization, the critical dynamics is significantly affected by the non-equilibrium conditions set by the microscopic theory. Specifically we show that all coherent dynamics, as measured by standard response functions, fades out at long wavelengths as a power-law with a new universal critical exponent. The decoherence exponent cannot be mimicked by any equilibrium model and places the critical dynamics of a driven system in a new dynamical universality class beyond the Halperin-Hohenberg classification of equilibrium dynamical critical behavior [14].

Open system dynamics- A microscopic description of driven open systems typically starts from a Markovian quantum master equation or an equivalent Keldysh action (see Supplementary Information (SI)). However, the novel aspects in the critical dynamics of driven dissipative systems discussed below can be most simply illustrated by considering an effective mesoscopic description of the order parameter dynamics using a stochastic Gross-Pitaevskii equation [15]

$$
i \partial_{t} \psi=\left[-(A-i D) \nabla^{2}-\mu+i \chi+(\lambda-i \kappa)|\psi|^{2}\right] \psi+\zeta .
$$

As we show below, this equation can be rigorously derived from a fully quantum microscopic description of the condensate when including only the relevant terms near the critical point. The different terms in (1) have a clear physical origin. $\chi=\left(\gamma_{p}-\gamma_{l}\right) / 2$ is the effective gain, which combines the incoherent pump field minus the local single-particle loss terms. $\kappa, \lambda>0$ are respectively two-body loss and interaction parameters. The diffusion term $D$ is not contained in the original microscopic model, and is not crucial to describe most nonuniversal aspects of, e.g., exciton-polariton condensates [16] (but see [17]). In a systematic treatment of long-wavelength universal critical behavior, however, such term is generated upon integrating out high frequency modes during the renormalization group (RG) flow, irrespective of its microscopic value. We therefore include it at the mesoscopic level with a phenomenological coefficient. Finally $\zeta$ is a Gaussian white noise with correlations $\left\langle\zeta^{*}(t, \mathbf{x}) \zeta\left(t^{\prime}, \mathbf{x}^{\prime}\right)\right\rangle=\gamma \delta\left(t-t^{\prime}\right) \delta\left(\mathbf{x}-\mathbf{x}^{\prime}\right)$ 
where $\gamma=\gamma_{p}+\gamma_{l}$. Such noise is necessarily induced by the losses and sudden appearances of particles due to pumping.

The dGP describes a mean field transition from a stationary condensate solution with density $|\psi|^{2}=\chi / \kappa$ for $\chi>0$ to the vacuum state when $\chi$ crosses zero. Dynamical stability [18] determines the chemical potential as $\mu=\lambda|\psi|^{2}$. Similar to a temperature, the noise term in Eq. (1) can drive a transition at finite particle density, thereby inducing critical fluctuations.

As the equation of motion is cast in Langevin form, one might suspect that it can be categorized into one of the wellknown models of dynamical critical phenomena classified by Hohenberg and Halperin [14]. However, this is not true in general. Crucially coherent (real parts of the couplings in Eq. (1)) and dissipative (imaginary parts) dynamics have different physical origins in driven-dissipative systems. In particular, the dissipative dynamics is determined by the intensity of the pump and loss terms, independently of the intrinsic Hamiltonian dynamics of the system. Equilibrium models [14], on the other hand, are constrained to have a specific relation between the reversible and dissipative terms to ensure a thermal Gibbs ensemble in steady state $[19,20]$ (see below). The unconstrained dynamics in driven systems is the key feature that can lead to novel dynamic critical behavior.

Microscopic Model - Having illustrated the nature of the problem with the effective classical equation (1) we turn to a fully quantum description within the Keldysh framework. Our starting point is a non-unitary quantum evolution described by a many-body master equation in Lindblad form, or equivalently by the following dissipative Keldysh action (see SI for details of the correspondence)

$$
\begin{aligned}
\mathcal{S}=\int_{t, \mathbf{x}}\left\{\left(\phi_{c}^{*}, \phi_{q}^{*}\right)\left(\begin{array}{cc}
0 & P^{A} \\
P^{R} & P^{K}
\end{array}\right)\left(\begin{array}{l}
\phi_{c} \\
\phi_{q}
\end{array}\right)+i 4 \kappa \phi_{c}^{*} \phi_{c} \phi_{q}^{*} \phi_{q}\right. \\
\left.-\left[(\lambda+i \kappa)\left(\phi_{c}^{* 2} \phi_{c} \phi_{q}+\phi_{q}^{* 2} \phi_{c} \phi_{q}\right)+c . c .\right]\right\} .
\end{aligned}
$$

Here $\phi_{c}, \phi_{q}$ are the "classical" and "quantum" fields, defined by the symmetric and anti-symmetric combinations of the fields on the forward and backward parts of the Keldysh contour [21, 22]. The microscopic inverse Green's functions are given by $P^{R}=i \partial_{t}+A \nabla^{2}+\mu-i \chi, P^{A}=P^{R^{\dagger}}, P^{K}=i \gamma$.

The importance of the various terms in the microscopic action (2) in the vicinity of the critical point can be inferred from canonical power counting, which serves as a valuable guideline for the explicit evaluation of the problem. Vanishing of the mass scale $\chi$ defines a Gaussian fixed point with dynamical critical exponent $z=2\left(\omega \sim k^{z}, k\right.$ a momentum scale). Canonical power counting determines the scaling dimensions of the fields and interaction constants with respect to this fixed point: At criticality, the spectral components of the Gaussian action scale as $P^{R / A} \sim k^{2}$, while the Keldysh component generically takes a constant value, i.e., $P^{K} \sim k^{0}$. Hence, to maintain scale invariance of the quadratic action, the scaling dimensions of the fields must be $\left[\phi_{c}\right]=\frac{d-2}{2}$ and $\left[\phi_{q}\right]=\frac{d+2}{2}$. From this result we read off the canonical scaling dimensions of the interaction constants. This analysis shows that in the case of interest $d=3$, local vertices containing more than two quantum fields or more than five classical fields are irrelevant. For the critical problem, the last terms in both lines of Eq. (2) can thus be skipped, massively simplifying the complexity of the problem. The only marginal term with two quantum fields is the Keldysh component of the singleparticle inverse Green's function, i.e., the noise vertex. In this sense, the critical theory is equivalent to a stochastic classical problem [23, 24], as previously observed in [8, 25]. But as noted above it cannot be a priori categorized in one of the dynamical universality classes [14] subject to an intrinsic equilibrium constraint.

Functional $R G$ - In order to focus quantitatively on the critical behavior we use a functional RG approach formulated originally by Wetterich [26] and adapted to the Keldysh real time framework in Refs. [27, 28] (see SI for details). At the formal level this technique provides an exact functional flow equation for an effective action functional $\Gamma_{\Lambda}\left[\phi_{c}, \phi_{q}\right]$, which includes information on increasingly long wavelength fluctuations (at the microscopic cutoff scale $\Gamma_{\Lambda_{0}} \approx \mathcal{S}$ ). In practice one works with an ansatz for the effective action and thereby projects the functional flow onto scaling equations for a finite set of coupling constants. For the description of general equilibrium [29-34] and Ising dynamical [35] critical behavior the functional RG gave results that are competitive with high-order epsilon expansion and with Monte Carlo simulations already in rather simple approximation schemes.

Our ansatz for the effective action is motivated by the power counting arguments introduced above. We include in $\Gamma_{\Lambda}$ all couplings that are relevant or marginal in this scheme:

$$
\Gamma_{\Lambda}=\int_{t, \mathbf{x}}\left\{\left(\phi_{c}^{*}, \phi_{q}^{*}\right)\left(\begin{array}{cc}
0 & i Z \partial_{t}+\bar{K} \nabla^{2} \\
i Z^{*} \partial_{t}+\bar{K}^{*} \nabla^{2} & i \bar{\gamma}
\end{array}\right)\left(\begin{array}{l}
\phi_{c} \\
\phi_{q}
\end{array}\right)-\left(\frac{\partial \bar{U}}{\partial \phi_{c}} \phi_{q}+\frac{\partial \bar{U}^{*}}{\partial \phi_{c}^{*}} \phi_{q}^{*}\right)\right\}
$$

The dynamical couplings $Z$ and $\bar{K}$ have to be taken complex valued in order to be consistent with power counting, even if the respective imaginary parts vanish (or are very small) at the microscopic scale: Successive momentum mode elimination implemented by the RG flow generates these terms due to the simultaneous presence of local coherent and dissipative couplings in the microscopic model. The fact that the spectral components of the effective action depend only linearly on $\phi_{q}$ allowed us to introduce an effective potential $\bar{U}$ determined by the complex static couplings. $\bar{U}\left(\rho_{c}\right)=\frac{1}{2} \bar{u}\left(\rho_{c}-\rho_{0}\right)^{2}+$ 
$\frac{1}{6} \bar{u}^{\prime}\left(\rho_{c}-\rho_{0}\right)^{3}$ is a function of the $U(1)$ invariant combination of classical fields $\rho_{c}=\phi_{c}^{*} \phi_{c}$ alone. It has a mexican hat structure ensuring dynamical stability. With this choice we approach the transition from the ordered side, taking the limit of the stationary state condensate $\rho_{0}=\left.\phi_{c}^{*} \phi_{c}\right|_{\mathrm{ss}}=\phi_{0}^{*} \phi_{0} \rightarrow 0$.

All the parameters appearing in (3) including the stationary condensate density $\rho_{0}$ are functions of the running cutoff $\Lambda$. Hence, the functional flow of $\Gamma_{\Lambda}$ is reduced by means of the approximate ansatz to the flow of a finite number of couplings $\mathbf{g}=\left(Z, \bar{K}, \rho_{0}, \bar{u}, \bar{u}^{\prime}, \bar{\gamma}\right)^{T}$ determined by the $\beta$-functions $\Lambda \partial_{\Lambda} \mathbf{g}=$ $\beta_{\mathrm{g}}(\mathrm{g})$ (see SI). The critical system is described by a scaling solution to these flow equations. It is obtained as a fixed point of the flow of dimensionless renormalized couplings, which we derive in the following. First we rescale couplings with $Z$,

$$
K=\bar{K} / Z, \quad u=\bar{u} / Z, \quad u^{\prime}=\bar{u}^{\prime} / Z, \quad \gamma=\bar{\gamma} /|Z|^{2} .
$$

Coherent and dissipative processes are encoded, respectively, in the real and imaginary parts of the renormalized coefficients $K=A+i D, u=\lambda+i \kappa$, and $u^{\prime}=\lambda^{\prime}+i \kappa^{\prime}$.

We define the first three dimensionless scaling variables to be the ratios of coherent to dissipative coefficients: $r_{K}=A / D$, $r_{u}=\lambda / \kappa$, and $r_{u^{\prime}}=\lambda^{\prime} / \kappa^{\prime}$. Another three dimensionless variables are defined by rescaling the loss coefficients $\kappa$ and $\kappa^{\prime}$ and the condensate density $\rho_{0}$ :

$$
w=\frac{2 \kappa \rho_{0}}{\Lambda^{2} D}, \quad \tilde{\kappa}=\frac{\gamma \kappa}{2 \Lambda D^{2}}, \quad \tilde{\kappa}^{\prime}=\frac{\gamma^{2} \kappa^{\prime}}{4 D^{3}} .
$$

The flow equations for the couplings $\mathbf{r}=\left(r_{K}, r_{u}, r_{u^{\prime}}\right)^{T}$ and $\mathbf{S}=\left(w, \tilde{\kappa}, \tilde{\kappa}^{\prime}\right)^{T}$ form a closed set,

$$
\Lambda \partial_{\Lambda} \mathbf{r}=\beta_{\mathbf{r}}(\mathbf{r}, \mathbf{s}), \quad \Lambda \partial_{\Lambda} \mathbf{s}=\beta_{\mathbf{s}}(\mathbf{r}, \mathbf{s})
$$

(see SI for the explicit form). As a consequence of the transformations (4) and (5), these $\beta$-functions acquire a contribution from the running anomalous dimensions $\eta_{a}(\mathbf{r}, \mathbf{s})=$ $-\Lambda \partial_{\Lambda} \ln a$ associated with $a=Z, D, \gamma$.

Critical properties - The universal behavior near the critical point is controlled by the infrared flow to a Wilson-Fisher like fixed point. The values of the coupling constants at the fixed point, determined by solving $\beta_{\mathbf{s}}\left(\mathbf{r}_{*}, \mathbf{s}_{*}\right)=0$ and $\beta_{\mathbf{r}}\left(\mathbf{r}_{*}, \mathbf{s}_{*}\right)=0$, are given by:

$$
\begin{aligned}
& \mathbf{r}_{*}=\left(r_{K_{*}}, r_{u^{*}}, r_{u^{\prime} *}\right)=\mathbf{0}, \\
& \mathbf{S}_{*}=\left(w_{*}, \tilde{\kappa}_{*}, \tilde{\kappa}_{*}^{\prime}\right) \approx(0.475,5.308,51.383) .
\end{aligned}
$$

The fact that $\mathbf{r}_{*}=0$ implies that the fixed point action is purely imaginary (or dissipative), as in Model A of Hohenberg and Halperin [14], cf. Fig. 1 (c). We interpret the fact that the ratios of coherent vs. dissipative couplings are zero at the fixed point as a manifestation of decoherence at low frequencies in an RG framework. The coupling values $\mathbf{s}_{*}$ are identical to those obtained in an equilibrium classical $O(2)$ model from functional RG calculations at the same level of truncation [29].

Let us turn to the linearized flow, which determines the universal behavior in the vicinity of the fixed point. We find that the two sectors corresponding to $\mathbf{s}$ and $\mathbf{r}$ decouple in this regime, giving rise to a block diagonal stability matrix

$$
\frac{\partial}{\partial \ln \Lambda}\left(\begin{array}{c}
\delta \mathbf{r} \\
\delta \mathbf{s}
\end{array}\right)=\left(\begin{array}{cc}
N & 0 \\
0 & S
\end{array}\right)\left(\begin{array}{c}
\delta \mathbf{r} \\
\delta \mathbf{s}
\end{array}\right),
$$

where $\delta \mathbf{r} \equiv \mathbf{r}, \delta \mathbf{s} \equiv \mathbf{s}-\mathbf{s}_{*}$, and $N, S$ are $3 \times 3$ matrices (see $\mathrm{SI}$ ).

The anomalous dimensions entering this flow are found by plugging the fixed point values $\mathbf{r}_{*}, \mathbf{s}_{*}$ into the expressions for $\eta_{a}(\mathbf{r}, \mathbf{s})$. We obtain the scaling relation between the anomalous dimensions $\eta_{Z}=\eta_{\bar{\gamma}}$, valid in the universal infrared regime. This leads to cancellation of $\eta_{Z}$ with $\eta_{\bar{\gamma}}$ in the static sector $S$ (see SI). The critical properties in this sector, encoded in the eigenvalues of $S$, become identical to those of the standard $O(2)$ transition. This includes the correlation length exponent $v \approx 0.716$ and the anomalous dimension $\eta \approx 0.039$ associated with the bare kinetic coefficient $\bar{K}$. These values are in good agreement with more sophisticated approximations [36].

The equilibrium-like behavior in the $S$ sector can be seen as a result of an emergent symmetry. Locking of the noise to the dynamical term implied by $\eta_{Z}=\eta_{\bar{\gamma}}$ leads to invariance of the long wavelength effective action (times $i$ ) under the transformation $\Phi_{c}(t, \mathbf{x}) \rightarrow \Phi_{c}(-t, \mathbf{x}), \Phi_{q}(t, \mathbf{x}) \rightarrow \Phi_{q}(-t, \mathbf{x})+$ $\frac{2}{\gamma} \sigma^{z} \partial_{t} \Phi_{c}(-t, \mathbf{x}), i \rightarrow-i$ with $\Phi_{v}=\left(\phi_{v}, \phi_{v}^{*}\right)^{T}, v=(c, q)$, $\sigma^{z}$ the Pauli matrix. It generalizes the symmetry noted in Refs. $[37,38]$ to models that include also reversible couplings. The presence of this symmetry implies a classical FDR with a distribution function $F=2 T_{\text {eff }} / \omega$, governed by an effective temperature $T_{\text {eff }}=\bar{\gamma} /(4|Z|)$. This quantity becomes scale independent in the universal critical regime where $\bar{\gamma} \sim k^{-\eta_{\bar{\gamma}}}$ and $Z \sim k^{-\eta_{Z}}$ cancel. We interpret this finding as an asymptotic low-frequency thermalization mechanism of the driven system at criticality. The thermalized regime sets in below the Ginzburg scale where fluctuations start to dominate, for which we estimate perturbatively $\chi_{G}=(\gamma \kappa)^{2} /\left(16 \pi^{2} D^{3}\right)$ (see SI). The values entering here are determined on the mesoscopic (a)

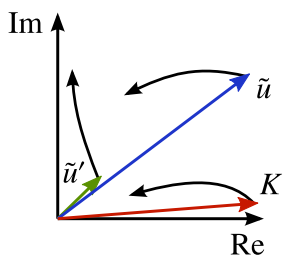

initial flow (b)

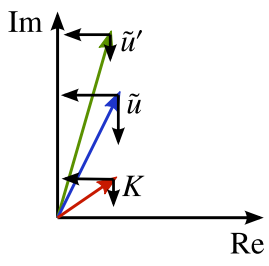

linearized IR flow (c)

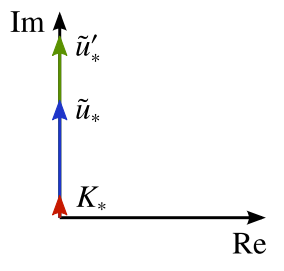

fixed point
Figure 1. Flow in the complex plane of dimensionless renormalized couplings. (a) The microscopic action determines the initial values of the flow. Typically, the coherent propagation will dominate over the diffusion, $A \gg D$, while two-body collisions and two-body loss are on the same order of magnitude, $\tilde{\lambda} \approx \tilde{\kappa}$, with a similar relation for the marginal complex coupling $\tilde{u}^{\prime}$. The initial flow is non-universal. (b) At criticality, the infrared (IR) flow approaches a universal linear domain encoding the critical exponents and anomalous dimensions. In particular, this regime is independent of the precise microscopic initial conditions. (c) The Wilson-Fisher fixed point describing the interacting critical system is purely imaginary. 
scale, and we specify them for exciton-polariton systems in the SI based on Ref. [17]. Above the scale $\chi_{G}$, no global (scale independent) temperature can be defined in general. We note that, unlike Hohenberg-Halperin type models, here the symmetry implied by $\eta_{Z}=\eta_{\bar{\gamma}}$ is not imposed at the microscopic level of the theory, but rather is emergent at the critical point.

The key new element in the driven-dissipative dynamics is encoded in the decoupled "drive" sector (the $3 \times 3$ matrix $N$ in our case). It describes the flow towards the emergent purely dissipative Model A fixed point (see Fig. 1 (b)) and thus reflects a mechanism of low frequency decoherence. This sector has no counterpart in the standard framework of dynamical critical phenomena and is special to driven-dissipative systems. In the deep infrared regime, only the lowest eigenvalue of this matrix governs the flow of the ratios. This means that only one new critical exponent $\eta_{r} \approx-0.101$ is encoded in this sector. Just as the dynamical critical exponent $z$ is independent of the static ones, the block diagonal structure of the stability matrix ensures that the drive exponent is independent of the exponents of the other sectors.

The fact that the inverse Green's function in Eq. (3) is specified by three real parameters, $\operatorname{Re} \bar{K}, \operatorname{Im} \bar{K}$, and $|Z|$ (the phase of $Z$ can be absorbed by a $U(1)$ transformation) allows for only three independent anomalous dimensions: $\eta_{D}, \eta_{Z}$ and the new exponent $\eta_{r}$. Hence the extension of critical dynamics described here is maximal, i.e., no further independent exponent will be found. Moreover this extension of the purely relaxational (Model A) dynamics leads to different universality than an extension that adds reversible couplings compatible with relaxation towards a Gibbs ensemble. The latter is obtained by adding real couplings to the imaginary ones with the same ratio of real to imaginary parts for all couplings [3942]; in this case the above symmetry is present, while absent in the general non-equilibrium case. The compatible extension adds only an independent $1 \times 1$ sector $N$ to the purely relaxational problem, for which we find $\eta_{R}=-0.143 \neq \eta_{r}$. This proves that the independence of dissipative and coherent dynamics defines indeed a new non-equilibrium universality class with no equilibrium counterpart. It is rooted in different symmetry properties of equilibrium vs. non-equilibrium situation.

Experimental detection - The novel anomalous dimension identified here leaves a clear fingerprint in single-particle observables accessible with current experimental technologies on different platforms. For ultracold atomic systems this can be achieved via RF-spectroscopy [43] close to the drivendissipative BEC transition. In exciton-polariton condensates, the dispersion relation can be obtained from the energy- and momentum resolved luminescence spectrum as demonstrated in [44]. Using the RG scaling behavior of the diffusion and propagation coefficients $D \sim D_{0} \Lambda^{-\eta_{D}}, A=D r_{K} \sim A_{0} \Lambda^{-\eta_{r}-\eta_{D}}$, we obtain the anomalous scaling of the frequency and momentum resolved, renormalized retarded Green's function $G^{R}(\omega, \mathbf{q})=\left(\omega-A_{0}|\mathbf{q}|^{2-\eta_{r}-\eta_{D}}+i D_{0}|\mathbf{q}|^{2-\eta_{D}}\right)^{-1}$, with $A_{0}$ and $D_{0}$ non-universal constants. Peak position and width are implied by the complex dispersion $\omega \approx A_{0}|\mathbf{q}|^{2.22}-i D_{0}|\mathbf{q}|^{2.12}$.
The energy resolution necessary to probe the critical behavior is again set by the Ginzburg scale $\chi_{G}$ (see above).

Conclusions - We have developed a Keldysh field theoretical approach to characterize the critical behavior of drivendissipative three dimensional Bose systems at the condensation transition. The main result presents a hierarchical extension of classical critical phenomena. First, all static aspects are identical to the classical $O(2)$ critical point. In the next shell of the hierarchy a sub-class of the dynamical phenomena is identical to the purely dissipative Model A dynamics of the equilibrium critical point. Finally we identify manifestly non-equilibrium features of the critical dynamics, encoded in a new independent critical exponent that betrays the driven nature of the system.

Acknowledgements - We thank J. Berges, M. Buchhold, I. Carusotto, T. Esslinger, T. Gasenzer, A. Imamoglu, J. M. Pawlowski, P. Strack, S. Takei, U. C. Täuber, C. Wetterich and P. Zoller for useful discussions. This research was supported by the Austrian Science Fund (FWF) through the START grant Y 581-N16 and the SFB FoQuS (FWF Project No. F4006-N16).

[1] I. Carusotto, C. Ciuti, Rev. Mod. Phys. 85, 299 (2013).

[2] J. Kasprzak et al., Nature 443, 409 (2006).

[3] R. J. Schoelkopf and S. M. Girvin, Nature 451, 7179 (2008).

[4] J. Clarke and F. K. Wilhelm, Nature 453, 1031 (2008).

[5] M. J. Hartmann, F. G. S. L. Brandao, M. B. Plenio, Laser \& Photon. Rev. 2, No. 6, 527 (2008).

[6] H. Ritsch, P. Domokos, F. Brennecke, T. Esslinger, arXiv:1210.0013 (2012).

[7] G. Roumpos et al., PNAS 109 no. 17, 6467 (2012).

[8] A. Mitra, S. Takei, Y. B. Kim and A. J. Millis, Phys. Rev. Lett. 97, 236808 (2006).

[9] S. Diehl et al., Nature Physics 4, 878 (2008); S. Diehl et al., Phys. Rev. Lett. 105, 015702 (2010).

[10] E. G. Dalla Torre, E. Demler, T. Giamarchi, E. Altman, Nature Physics 6, 806 (2010).

[11] E. G. Dalla Torre et al., Phys. Rev. A 87, 023831 (2013).

[12] B. Öztop, M. Bordyuh, O. E. Müstecaplioglu, and H. E. Türeci, New J. Phys. 14, 085011 (2012).

[13] M. Wouters and I. Carusotto, Physical Review B 74, 245316 (2006).

[14] P. C. Hohenberg and B. I. Halperin, Rev. Mod. Phys. 49, 435 (1977).

[15] I. Carusotto and C. Ciuti, Physical Review B 72, 125335 (2005).

[16] M. Wouters and I. Carusotto, Phys. Rev. Lett. 99, 140402 (2007); J. Keeling, P. R. Eastham, M. H. Szymanska, and P. B. Littlewood, Phys. Rev. Lett. 93, 226403 (2004); M. H. Szymanska, J. Keeling, and P. B. Littlewood, Phys. Rev. Lett. 96, 230602 (2006); J. Keeling and N.G. Berloff, Phys. Rev. Lett 100, 250401 (2008).

[17] M. Wouters and I. Carusotto, Phys. Rev. Lett. 105, 020602 (2010); M. Wouters, T. C. H. Liew, and V. Savona, Phys. Rev. B 82, 245315 (2010).

[18] J. Keeling, M. H. Szymanska, P. B. Littlewood, in Optical Generation and Control of Quantum Coherence in Semiconduc- 
tor Nanostructures, Nanoscience and Technology, edited by G. Slavcheva and P. Roussignol (Springer Berlin, 2010) pp. 293329.

[19] P. M. Chaikin and T. C. Lubensky, Principles of Condensed Matter Physics, (Cambridge University Press, 1995)

[20] U. C. Täuber, Lecture Notes Phys. 716295 (2007).

[21] A. Kamenev and A. Levchenko, Advances in Physics, 58(3), 197 (2009).

[22] A. Altland and B. Simons, Condensed Matter Field Theory (Cambridge University Press, 2010).

[23] P. C. Martin, E. D. Siggia, H. A. Rose, Phys. Rev. A 8, 423 (1973).

[24] J. DeDominics, J. Physique (Paris) 37, C1 (1976).

[25] A. Mitra, A. Rosch, Phys. Rev. Lett. 106, 106402 (2011).

[26] C. Wetterich, Phys. Lett. B 301, 90 (1993); Z. Phys. C 57, 451 (1993).

[27] T. Gasenzer, J. M. Pawlowski, Phys. Lett. B 670135 (2008).

[28] J. Berges, G. Hoffmeister, Nucl. Phys. B 813383 (2009).

[29] J. Berges, N. Tetradis, C. Wetterich, Phys. Rept. 363223 (2002).

[30] M. Salmhofer and C. Honerkamp, Prog. Theor. Phys. 105, 1 (2001).
[31] J. M. Pawlowski, Annals Phys. 322, 2831 (2007).

[32] B. Delamotte, cond-mat/0702365 (2007).

[33] O. J. Rosten, Physics Reports 511, 177 (2012).

[34] I. Boettcher, J. M. Pawlowski, S. Diehl, Nucl. Phys. Proc. Suppl. 228, 63 (2012).

[35] L. Canet and H. Chaté J. Phys. A: Math. Theor. 40, 1937 (2007).

[36] R. Guida and J. Zinn-Justin, J. Phys. A 31, 8103 (1998).

[37] C. Aron, G. Biroli, and L. F. Cugliandolo, J. Stat. Mech. 1011, 11018 (2010).

[38] L. Canet, H. Chaté, B. Delamotte, J. Phys. A: Math. Theor. 44, 495001 (2011).

[39] L. M. Sieberer, S. D. Huber, E. Altman, S. Diehl, in preparation (2013).

[40] R. Graham, Springer Tracts in Modern Physics, Vol. 66 (Springer-Verlag, Berlin, 1973).

[41] U. Deker and F. Haake, Phys. Rev. A 11, 2043 (1975).

[42] U. C. Täuber, V. K. Akkineni, J. E. Santos, Phys. Rev. Lett. 88 045702 (2002).

[43] J. T. Stewart, J. P. Gaebler, and D. S. Jin, Nature 454, 744 (2008).

[44] S. Utsunomiya et al., Nature Phys. 4, 700 (2008). 


\title{
Supplementary Information for "Dynamical Critical Phenomena in Driven-Dissipative Systems"
}

\author{
L. M. Sieberer ${ }^{1,2}$, S. D. Huber ${ }^{3,4}$, E. Altman ${ }^{4,5}$, and S. Diehl ${ }^{1,2}$ \\ ${ }^{1}$ Institute for Theoretical Physics, University of Innsbruck, A-6020 Innsbruck, Austria \\ ${ }^{2}$ Institute for Quantum Optics and Quantum Information of the Austrian Academy of Sciences, A-6020 Innsbruck, Austria \\ ${ }^{3}$ Theoretische Physik, Wolfgang-Pauli-Strasse 27, ETH Zurich, CH-8093 Zurich, Switzerland \\ ${ }^{4}$ Department of Condensed Matter Physics, Weizmann Institute of Science, Rehovot 76100, Israel \\ ${ }^{5}$ Department of Physics, University of California, Berkeley, CA 94720, USA
}

\section{OPEN SYSTEM DYNAMICS}

Open system dynamics with local particle loss and gain can be modeled microscopically by a many-body quantum master equation $(\hbar=1)$

$$
\partial_{t} \hat{\rho}=-i[\hat{H}, \hat{\rho}]+\mathcal{L}[\hat{\rho}]
$$

The dynamics of the system density matrix $\hat{\rho}$ has both a coherent contribution due to the standard Hamiltonian for bosons of $\operatorname{mass} m\left(\int_{\mathbf{x}}=\int d^{3} \mathbf{x}\right)$

$$
\hat{H}=\int_{\mathbf{x}} \hat{\psi}^{\dagger}(\mathbf{x})\left(-\frac{\Delta}{2 m}-\mu\right) \hat{\psi}(\mathbf{x})+\lambda \int_{\mathbf{x}} \hat{\psi}^{\dagger}(\mathbf{x})^{2} \hat{\psi}(\mathbf{x})^{2},
$$

and a dissipative one that is incorporated by the Liouville operator

$$
\begin{aligned}
\mathcal{L}[\hat{\rho}]= & \gamma_{p} \int_{\mathbf{x}}\left(\hat{\psi}^{\dagger}(\mathbf{x}) \hat{\rho} \hat{\psi}(\mathbf{x})-\frac{1}{2}\left\{\hat{\psi}(\mathbf{x}) \hat{\psi}^{\dagger}(\mathbf{x}), \hat{\rho}\right\}\right) \\
& +\gamma_{l} \int_{\mathbf{x}}\left(\hat{\psi}(\mathbf{x}) \hat{\rho} \hat{\psi}^{\dagger}(\mathbf{x})-\frac{1}{2}\left\{\hat{\psi}^{\dagger}(\mathbf{x}) \hat{\psi}(\mathbf{x}), \hat{\rho}\right\}\right) \\
+ & 2 \kappa \int_{\mathbf{x}}\left(\hat{\psi}(\mathbf{x})^{2} \hat{\rho} \hat{\psi}^{\dagger}(\mathbf{x})^{2}-\frac{1}{2}\left\{\hat{\psi}^{\dagger}(\mathbf{x})^{2} \hat{\psi}(\mathbf{x})^{2}, \hat{\rho}\right\}\right) .
\end{aligned}
$$

Local Lindblad operators $\hat{\psi}^{\dagger}(\mathbf{x})$ and $\hat{\psi}(\mathbf{x})$, respectively, correspond to the processes of incoherent pumping and loss of single particles; $\hat{\psi}(\mathbf{x})^{2}$ describes the simultaneous loss of two particles. Associated rates are $\gamma_{p}, \gamma_{l}$, and $2 \kappa$.

The investigation of critical phenomena at the stationary state phase transition exhibited by this model is facilitated by a formulation in terms of a Keldysh partition function [1,2] $\mathcal{Z}=\int \mathcal{D} \psi_{+} \mathcal{D} \psi_{-} e^{i S}$, which can be subject to renormalization group methods. This partition function is fully equivalent to the master equation (SI-1) and defined in terms of a Keldysh action $\mathcal{S}=\mathcal{S}_{H}+\mathcal{S}_{D}$ with two contributions corresponding to the commutator with the Hamiltonian (from now on we will be using units such that $2 m=1 ; \int_{t, \mathbf{x}}=\int d t \int d \mathbf{x}$ ),

$$
\mathcal{S}_{H}=\sum_{\sigma= \pm} \sigma \int_{t, \mathbf{x}}\left[\psi_{\sigma}^{*}\left(i \partial_{t}+\Delta+\mu\right) \psi_{\sigma}-\lambda\left(\psi_{\sigma}^{*} \psi_{\sigma}\right)^{2}\right]
$$

and the dissipative Liouvillian,

$$
\begin{gathered}
\mathcal{S}_{D}=-i \gamma_{p} \int_{t, \mathbf{x}}\left[\psi_{+}^{*} \psi_{-}-\frac{1}{2}\left(\psi_{+} \psi_{+}^{*}+\psi_{-} \psi_{-}^{*}\right)\right] \\
-i \gamma_{l} \int_{t, \mathbf{x}}\left[\psi_{+} \psi_{-}^{*}-\frac{1}{2}\left(\psi_{+}^{*} \psi_{+}+\psi_{-}^{*} \psi_{-}\right)\right] \\
-i 2 \kappa \int_{t, \mathbf{x}}\left\{\left(\psi_{+} \psi_{-}^{*}\right)^{2}-\frac{1}{2}\left[\left(\psi_{+}^{*} \psi_{+}\right)^{2}+\left(\psi_{-}^{*} \psi_{-}\right)^{2}\right]\right\} .
\end{gathered}
$$

Expressing the Keldysh action in terms of classical and quantum fields, which are defined as

$$
\phi_{c}=\frac{1}{\sqrt{2}}\left(\psi_{+}+\psi_{-}\right), \quad \phi_{q}=\frac{1}{\sqrt{2}}\left(\psi_{+}-\psi_{-}\right),
$$

we recover Eq. (2) of the main text.

\section{FUNCTIONAL RENORMALIZATION GROUP EQUATION}

Our approach to studying critical dynamics is based on the Wetterich functional renormalization group [3] adapted to the Keldysh framework (see [4-9] for reviews on the equilibrium formulation). Central to this method is the functional $\Gamma_{\Lambda}\left[\phi_{c}, \phi_{q}\right]$ defined by [10]

$$
e^{i \Gamma_{\Lambda}\left[\phi_{c}, \phi_{q}\right]}=\int \mathcal{D} \delta \phi_{c} \mathcal{D} \delta \phi_{q} e^{i \mathcal{S}\left[\phi_{c}+\delta \phi_{c}, \phi_{q}+\delta \phi_{q}\right]+i \Delta \mathcal{S}_{\Lambda}\left[\delta \phi_{c}, \delta \phi_{q}\right]} .
$$

Here $\Delta \mathcal{S}_{\Lambda}$ is a regulator function which suppresses contributions to the above path integral from modes with spatial wavevector below the running cutoff $\Lambda$. Thus $\Gamma_{\Lambda}$ interpolates between the classical action $\mathcal{S}$, when $\Lambda$ equals the UV cutoff $\Lambda_{0}$, and the effective action functional $\Gamma\left[\phi_{c}, \phi_{q}\right][11]$ when $\Lambda \rightarrow 0$. The latter includes the effects of fluctuations on all scales. The equation

$$
\partial_{\Lambda} \Gamma_{\Lambda}=\frac{i}{2} \operatorname{Tr}\left[\left(\Gamma_{\Lambda}^{(2)}+R_{\Lambda}\right)^{-1} \partial_{\Lambda} R_{\Lambda}\right]
$$

describes the flow of the interpolating functional as a function of the running cutoff $\Lambda$. In the following sections we first discuss the objects appearing in (SI-8), namely the second functional derivative $\Gamma_{\Lambda}^{(2)}$ and the cutoff function $R_{\Lambda}$. Then we explain how a closed set of flow equations for a finite number of coupling constants can be obtained from the functional flow 
equation. Finally we detail the linearized equations for the infrared flow to the Wilson-Fisher fixed point from which the critical properties are inferred.

In suitable truncation schemes, results from high order epsilon expansion can be reproduced from the exact flow equation (SI-8). In our practical calculation, we approach the critical point from the ordered phase. This allows us to calculate the anomalous dimensions at one-loop order, due to the presence of a finite condensate during the flow. Results obtained in this way have proven to be competitive with high-order epsilon expansion or Monte Carlo simulations, as referenced in the main text.

\section{THE SECOND VARIATIONAL DERIVATIVE}

The second variation $\Gamma_{\Lambda}^{(2)}$ with respect to the fields is the full inverse Green's function at the scale $\Lambda$, which in the case of an interacting theory is field dependent. Practically we work in a basis of real fields, related to the complex fields by

$$
\left(\begin{array}{l}
\chi_{v, 1}(Q) \\
\chi_{v, 2}(Q)
\end{array}\right)=\frac{1}{\sqrt{2}}\left(\begin{array}{cc}
1 & 1 \\
-i & i
\end{array}\right)\left(\begin{array}{c}
\phi_{v}(Q) \\
\phi_{v}^{*}(-Q)
\end{array}\right)
$$

where $v=c, q$ is the Keldysh index. We gather the resulting four independent field components in a field vector,

$$
\chi(Q)=\left(\chi_{c, 1}(Q), \chi_{c, 2}(Q), \chi_{q, 1}(Q), \chi_{q, 2}(Q)\right)^{T} .
$$

In this basis, $\Gamma_{\Lambda}^{(2)}$ is defined as

$$
\left(\Gamma_{\Lambda}^{(2)}\right)_{i j}\left(Q, Q^{\prime}\right)=\frac{\delta^{2} \Gamma_{\Lambda}}{\delta \chi_{i}(-Q) \delta \chi_{j}\left(Q^{\prime}\right)},
$$

which is a matrix in the discrete field index $i=1,2,3,4$ and in the continuous momentum variable $Q=(\omega, \mathbf{q})$ collecting frequency and spatial momentum. Accordingly, the trace in (SI-8) involves both an integration over momenta and a sum over internal indices.

$\Gamma_{\Lambda}^{(2)}\left(Q, Q^{\prime}\right)$ is conveniently decomposed into a constant part and a fluctuation part. The latter is a polynomial in momentum-dependent fields and, therefore, a non-diagonal matrix in momentum space. In contrast, the constant part is obtained by (i) inserting spatially constant field configurations, i.e., $\chi(Q)=\chi \delta(Q)$ in momentum space, and (ii) evaluating them at their stationary state values in the ordered phase. These read

$$
\left.\chi(Q)\right|_{\mathrm{ss}}=\left(\sqrt{2 \rho_{0}}, 0,0,0\right)^{T} \delta(Q) .
$$

(Without loss of generality we choose the condensate amplitude to be real.) As a result, the constant part is diagonal in momentum space,

$$
\left.P_{\Lambda}(Q) \delta\left(Q-Q^{\prime}\right) \equiv \Gamma_{\Lambda}^{(2)}\left(Q, Q^{\prime}\right)\right|_{\mathrm{ss}},
$$

and is structured into retarded, advanced, and Keldysh blocks,

$$
P_{\Lambda}(Q)=\left(\begin{array}{cc}
0 & P^{A}(Q) \\
P^{R}(Q) & P^{K}
\end{array}\right) .
$$

(For notational simplicity, we suppress the scale index $\Lambda$ for the different blocks and their respective entries.) The retarded and advanced blocks are mutually hermitian conjugate (we decompose $Z$ and $\bar{K}$ into real and imaginary parts, $Z=Z_{R}+$ $\left.i Z_{I}, \bar{K}=\bar{A}+i \bar{D}\right)$,

$$
\begin{aligned}
& P^{R}(Q)=\left(\begin{array}{lc}
-i Z_{I} \omega-\bar{A} \mathbf{q}^{2}-2 \operatorname{Re}(\bar{u}) \rho_{0} & i Z_{R} \omega-\bar{D} \mathbf{q}^{2} \\
-i Z_{R} \omega+\bar{D} \mathbf{q}^{2}+2 \operatorname{Im}(\bar{u}) \rho_{0} & -i Z_{I} \omega-\bar{A} \mathbf{q}^{2}
\end{array}\right), \\
& P^{A}(Q)=\left(P^{R}(Q)\right)^{\dagger} .
\end{aligned}
$$

Note that $\operatorname{det} P^{R}(Q=0)=\operatorname{det} P^{A}(Q=0)=0$; the existence of a gapless mode associated to the broken $U(1)$ symmetry is thus ensured in our truncation at all scales $\Lambda$. For the Keldysh block we have

$$
P^{K}=i \bar{\gamma} \mathbb{1}
$$

\section{THE REGULATOR FUNCTION}

The cutoff contribution $\Delta \mathcal{S}_{\Lambda}$ is used in Eq. (SI-7) to generate the effective action $\Gamma_{\Lambda}$ from the microscopic action $\mathcal{S}$ by suppressing contributions from momenta below $\Lambda$. Its second functional derivative $R_{\Lambda}=\Delta \mathcal{S}_{\Lambda}^{(2)}$ enters the exact flow equation (SI-8). We choose an optimized cutoff function [12] of the form

$$
R_{\Lambda}(Q)=\left(\mathbf{q}^{2}-\Lambda^{2}\right) \theta\left(\Lambda^{2}-\mathbf{q}^{2}\right)\left(\begin{array}{cc}
0 & R^{R} \\
R^{A} & 0
\end{array}\right),
$$

where

$$
R^{R}=\left(\begin{array}{cc}
-\bar{A} & -\bar{D} \\
\bar{D} & -\bar{A}
\end{array}\right), \quad R^{A}=\left(R^{R}\right)^{T} .
$$

Due to the $\theta$-function in (SI-17), in the regularized inverse Green's function

$$
G_{\Lambda}^{-1}=P_{\Lambda}+R_{\Lambda},
$$

momenta $\mathbf{q}^{2}$ smaller than the running scale $\Lambda^{2}$ acquire an effective mass $\propto \Lambda^{2}$ and we have $\operatorname{det} G_{\Lambda}^{-1}(Q=0) \neq 0$, which ensures that momentum integrals over Green's functions are infrared convergent. Note that it is sufficient for $R_{\Lambda}$ to modify only the retarded and advanced blocks (i.e., the spectrum) of the inverse Green's function. The choice of a frequencyindependent cutoff allows us to perform frequency integrals analytically.

The interpolation property of $\Gamma_{\Lambda}$ between the classical action $\mathcal{S}$ and the effective action $\Gamma$ is guaranteed by the limiting behavior [13]

$$
\lim _{\Lambda^{2} \rightarrow \Lambda_{0}^{2}} R_{\Lambda} \sim \Lambda_{0}^{2}, \quad \lim _{\Lambda^{2} \rightarrow 0} R_{\Lambda}=0
$$

\section{FLOW OF THE EFFECTIVE POTENTIAL}

In equilibrium problems, an important object for practical calculations is the effective potential. It describes the homogeneous part of the effective action and is obtained by evaluating the full effective action at spatially homogeneous field 
configurations, $\bar{U}=\Gamma /\left.\Omega\right|_{\chi(Q)=\chi \delta(Q)}(\Omega$ is the quantization volume). In the framework of a derivative expansion, a closed flow equation can be derived for this object, which serves as a compact generating functional for the flow of all local couplings to arbitrarily high order. Here we provide the Keldysh analog of this construction, where the key difference roots in the occurrence of two field variables $\phi_{c}, \phi_{q}$, in contrast to a single field in equilibrium. However, for a theory which obeys the power counting discussed in the main text, we can parameterize the homogeneous part of the effective action as

$$
\bar{V}=\frac{\partial \bar{U}}{\partial \phi_{c}} \phi_{q}+\frac{\partial \bar{U}^{*}}{\partial \phi_{c}^{*}} \phi_{q}^{*}+i \bar{\gamma} \phi_{q}^{*} \phi_{q},
$$

with $\bar{U}=\bar{U}\left(\phi_{c}^{*} \phi_{c}\right)$ dependent on the $U(1)$ invariant combination of classical fields only, this function thus being the direct counterpart of the effective potential. A flow equation can be derived for the auxiliary object $\bar{V}$, which reads (we introduce a dimensionless scale derivative $\partial_{\ell} \equiv \Lambda \partial_{\Lambda}$ )

$$
\partial_{\ell} \bar{V}=-\frac{i}{2} \int_{Q} \operatorname{tr}\left[\mathcal{G}_{\Lambda}(Q) \partial_{\ell} R_{\Lambda}(Q)\right] .
$$

Here, the inverse of $\mathcal{G}_{\Lambda}$ is obtained from the full second functional variation by evaluating it at homogeneous field configurations (step (i) above Eq. (SI-12)), however without inserting the stationary state values (step (ii)): $\mathcal{G}_{\Lambda}^{-1}=\left.\Gamma_{\Lambda}^{(2)}\right|_{\chi(Q)=\chi \delta(Q)}+R_{\Lambda}$. $\mathcal{G}_{\Lambda}$ is then diagonal in momentum space, and so the trace in Eq. (SI-8) reduces to a single momentum integration, giving rise to the above compact form. In contrast to $G_{\Lambda}^{-1}, \mathcal{G}_{\Lambda}^{-1}$ has a non-vanishing upper left block $P^{H}$. However, it vanishes when the background fields are set to their stationary state values, $\left.P^{H}\right|_{\mathrm{ss}}=0$, which is a manifestation of causality in the Keldysh formalism [1, 2].

From this equation we obtain the $\beta$-functions for the momentum-independent couplings by evaluating appropriate derivatives with respect to the $U(1)$ invariants

$$
\rho_{c}=\phi_{c}^{*} \phi_{c}, \quad \rho_{c q}=\phi_{c}^{*} \phi_{q}=\rho_{q c}^{*}, \quad \rho_{q}=\phi_{q}^{*} \phi_{q} .
$$

at their stationary state values $\left.\rho_{c}\right|_{\mathrm{ss}}=\rho_{0},\left.\rho_{c q}\right|_{\mathrm{ss}}=\left.\rho_{q c}\right|_{\mathrm{ss}}=$ $\left.\rho_{q}\right|_{\mathrm{ss}}=0$. Specifically, we use the projection prescriptions

$$
\begin{aligned}
\partial_{\ell} \rho_{0} & =\beta_{\rho_{0}}=-\frac{1}{u}\left[\partial_{\rho_{c q}} \partial_{\ell} \bar{V}\right]_{\mathrm{ss}}, \\
\partial_{\ell} \bar{u} & =\beta_{\bar{u}}=\bar{u}^{\prime} \partial_{\ell} \rho_{0}+\left[\partial_{\rho_{c} \rho_{c q}}^{2} \partial_{\ell} \bar{V}\right]_{\mathrm{ss}}, \\
\partial_{\ell} \bar{u}^{\prime} & =\beta_{\bar{u}^{\prime}}=\left[\partial_{\rho_{c}}^{2} \partial_{\rho_{c q}} \partial_{\ell} \bar{V}\right]_{\mathrm{ss}}, \\
\partial_{\ell} \bar{\gamma} & =\beta_{\bar{\gamma}}=i \rho_{0}\left[\partial_{\rho_{c q} \rho_{q c}}^{2} \partial_{\ell} \bar{V}\right]_{\mathrm{ss}} .
\end{aligned}
$$

Calculation of the explicit expressions here and below is largely automatized using MathEMATICA.

\section{FLOW OF THE INVERSE PROPAGATOR}

While the flow equation for the effective potential (SI-22) generates $\beta$-functions for all momentum-independent couplings, the flow of the complex dynamic $Z$ and kinetic $\bar{K}$ couplings, which constitute the momentum-dependent part of the effective action (3), is determined by the flow equation for the inverse propagator. We obtain the latter by taking the second variational derivative of the exact flow equation (SI-8) and setting the background fields to their stationary state values Eq. (SI-12),

$$
\begin{aligned}
\partial_{\ell} P_{\Lambda, i j}(Q)= & \\
\frac{i}{2} \int_{Q^{\prime}} \operatorname{tr}[ & {\left[G_{\Lambda}^{2}\left(Q^{\prime}-Q\right) \partial_{\ell} R_{\Lambda}\left(Q^{\prime}-Q\right) \gamma_{i} G_{\Lambda}\left(Q^{\prime}\right) \gamma_{j}\right.} \\
& \left.+G_{\Lambda}\left(Q^{\prime}-Q\right) \gamma_{i} G_{\Lambda}^{2}\left(Q^{\prime}\right) \partial_{\ell} R_{\Lambda}\left(Q^{\prime}\right) \gamma_{j}\right],
\end{aligned}
$$

where

$$
\gamma_{i, j l} \delta\left(P-P^{\prime}+Q\right)=\left.\frac{\delta \Gamma_{\Lambda, j l}^{(2)}\left(P, P^{\prime}\right)}{\delta \chi_{i}(Q)}\right|_{\mathrm{ss}}
$$

In Eq. (SI-25) we omit tadpole contributions $\propto \Gamma_{\Lambda}^{(4)}$, which do not depend on the external momentum $Q$ and hence do not contribute to the flow of $Z$ or $\bar{K}$. For these we use the projection prescriptions

$$
\begin{aligned}
& \partial_{\ell} Z=\beta_{Z}=-\left.\frac{1}{2} \partial_{\omega} \operatorname{tr}\left[\left(\mathbb{1}+\sigma_{y}\right) \partial_{\ell} P^{R}(Q)\right]\right|_{Q=0}, \\
& \partial_{\ell} \bar{K}=\beta_{\bar{K}}=\left.\partial_{\mathbf{q}^{2}}\left[\partial_{\ell} P_{22}^{R}(Q)+i \partial_{\ell} P_{12}^{R}(Q)\right]\right|_{Q=0} .
\end{aligned}
$$

The $\beta$-functions (SI-24) and (SI-27) constitute the components of $\beta_{\mathbf{g}}=\left(\beta_{Z}, \beta_{\bar{K}}, \beta_{\rho_{0}}, \beta_{\bar{u}}, \beta_{\bar{u}^{\prime}}, \beta_{\bar{\gamma}}\right)^{T}$.

\section{RESCALED FLOW EQUATIONS}

We write the flow equation for the complex dynamic coupling $Z$ in the form

$$
\partial_{\ell} Z=-\eta_{Z} Z
$$

The anomalous dimension $\eta_{Z}$ is an algebraic function of the rescaled couplings (6) and $\rho_{0}$. The same applies to the $\beta$ functions of the latter,

$$
\begin{aligned}
& \partial_{\ell} K=\beta_{K}=\eta_{Z} K+\frac{1}{Z} \beta_{\bar{K}}, \\
& \partial_{\ell} u=\beta_{u}=\eta_{Z} u+\frac{1}{Z} \beta_{\bar{u}}, \\
& \partial_{\ell} u^{\prime}=\beta_{u^{\prime}}=\eta_{Z} u^{\prime}+\frac{1}{Z} \beta_{\bar{u}^{\prime}}, \\
& \partial_{\ell} \gamma=\beta_{\gamma}=\left(\eta_{Z}+\eta_{Z}^{*}\right) \gamma+\frac{1}{|Z|^{2}} \beta_{\bar{\gamma}} .
\end{aligned}
$$

In particular, the very right expressions in these equations $\left(\beta_{\bar{K}} / Z\right.$ etc.) are functions of the rescaled couplings alone. In terms of these variables, therefore, all explicit reference to the running coupling $Z$ is gone, and we have effectively traded the differential flow equation for $Z$ for the algebraic expression for its anomalous dimension $\eta_{Z}$. 
All couplings except for $\gamma$ are complex valued. Taking real and imaginary parts of the $\beta$-functions for $K, u$, and $u^{\prime}$ yields the flow equations for $A, D, \lambda, \kappa, \lambda^{\prime}$, and $\kappa^{\prime}$ respectively,

$$
\begin{aligned}
\partial_{\ell} A & =\beta_{A}=\operatorname{Re} \beta_{K}, & \partial_{\ell} D & =\beta_{D}=\operatorname{Im} \beta_{K}, \\
\partial_{\ell} \lambda & =\beta_{\lambda}=\operatorname{Re} \beta_{u}, & \partial_{\ell} \kappa & =\beta_{\kappa}=\operatorname{Im} \beta_{u}, \\
\partial_{\ell} \lambda^{\prime} & =\beta_{\lambda^{\prime}}=\operatorname{Re} \beta_{u^{\prime}}, & \partial_{\ell} \kappa^{\prime} & =\beta_{\kappa^{\prime}}=\operatorname{Im} \beta_{u^{\prime}} .
\end{aligned}
$$

The $\beta$-functions for the ratios $\mathbf{r}=\left(r_{K}, r_{u}, r_{u^{\prime}}\right)^{T}$ are then

$$
\begin{aligned}
& \partial_{\ell} r_{K}=\beta_{r_{K}}=\frac{1}{D} \beta_{A}-\frac{r_{K}}{D} \beta_{D}, \\
& \partial_{\ell} r_{u}=\beta_{r_{u}}=\frac{1}{\kappa} \beta_{\lambda}-\frac{r_{u}}{\kappa} \beta_{\kappa}, \\
& \partial_{\ell} r_{u^{\prime}}=\beta_{r_{u^{\prime}}}=\frac{1}{\kappa^{\prime}} \beta_{\lambda^{\prime}}-\frac{r_{u^{\prime}}}{\kappa^{\prime}} \beta_{\kappa^{\prime}} .
\end{aligned}
$$

The number of flow equations can be further reduced by introducing anomalous dimensions for $D$ and $\gamma$,

$$
\begin{gathered}
\partial_{\ell} D=-\eta_{D} D, \\
\partial_{\ell} \gamma=-\eta_{\gamma} \gamma .
\end{gathered}
$$

As for the dynamic coupling $Z$ in terms of the rescaled variables $K, u, u^{\prime}, \gamma$ and $\rho_{0}$, all explicit reference to $D$ and $\gamma$ drops out, and we obtain for the couplings $\mathbf{S}=\left(w, \tilde{\kappa}, \tilde{\kappa}^{\prime}\right)^{T}$ defined in Eq. (5)

$$
\begin{gathered}
\partial_{\ell} w=\beta_{w}=-\left(2-\eta_{D}\right) w+\frac{w}{\kappa} \beta_{\kappa}+\frac{2 \kappa}{\Lambda^{2} D} \beta_{\rho_{0}}, \\
\partial_{\ell} \tilde{\kappa}=\beta_{\tilde{\kappa}}=-\left(1-2 \eta_{D}+\eta_{\gamma}\right) \tilde{\kappa}+\frac{\gamma}{2 \Lambda D^{2}} \beta_{\kappa}, \\
\partial_{\ell} \tilde{\kappa}^{\prime}=\beta_{\tilde{\kappa}^{\prime}}=-\left(-3 \eta_{D}+2 \eta_{\gamma}\right) \tilde{\kappa}^{\prime}+\frac{\gamma^{2}}{4 D^{3}} \beta_{\kappa^{\prime}} .
\end{gathered}
$$

In summary, the transformations (4) and (5) result in the closed system (6) for $\mathbf{r}$ and $\mathbf{s}$ with $\beta_{\mathbf{r}}=\left(\beta_{r_{K}}, \beta_{r_{u}}, \beta_{r_{u^{\prime}}}\right)^{T}$ given by Eq. (SI-31) and $\beta_{\mathrm{S}}=\left(\beta_{w}, \beta_{\tilde{K}}, \beta_{\tilde{K}^{\prime}}\right)^{T}$ given by Eq. (SI-33). The flows of $Z, D$, and $\gamma$ are decoupled and determined by the anomalous dimensions (SI-28) and (SI-32), which are themselves functions of $\mathbf{r}$ and $\mathbf{s}$.

\section{CRITICAL PROPERTIES}

For the analysis of critical behavior, we need to find a scaling solution to the flow equations for the bare couplings or, equivalently, a fixed point $\mathbf{r}_{*}, \mathbf{s}_{*}$ of the flow of dimensionless rescaled couplings,

$$
\beta_{\mathbf{r}}\left(\mathbf{r}_{*}, \mathbf{S}_{*}\right)=\beta_{\mathbf{s}}\left(\mathbf{r}_{*}, \mathbf{S}_{*}\right)=\mathbf{0} .
$$

This non-linear algebraic set of equations has a non-trivial solution given by Eq. (7). In order to characterize the infrared flow in the vicinity of the fixed point (encoding the critical exponents we are interested in here), we study the flow of the couplings linearized around the fixed point, cf. Eq. (8). The stability matrices $N$ and $S$ in this equation read explicitly

$$
\begin{gathered}
N=\left.\nabla_{\mathbf{r}}^{T} \beta_{\mathbf{r}}\right|_{\mathbf{r}=\mathbf{r}_{*}, \mathbf{s}=\mathbf{s}_{*}}=\left(\begin{array}{ccc}
0.0525 & 0.0586 & 0.0317 \\
-0.0002 & -0.0526 & 0.1956 \\
0.4976 & -2.3273 & 1.9725
\end{array}\right), \\
S=\left.\nabla_{\mathbf{s}}^{T} \beta_{\mathbf{s}}\right|_{\mathbf{r}=\mathbf{r}_{*}, \mathbf{s}=\mathbf{s}_{*}}=\left(\begin{array}{ccc}
-1.6204 & 0.0881 & 0.0046 \\
-3.1828 & 0.2899 & 0.0363 \\
-15.3743 & -42.2487 & 2.1828
\end{array}\right),
\end{gathered}
$$

without coupling between $\mathbf{r}$ and $\mathbf{s}$ sectors. At present we cannot rule out that an extended truncation would couple them. However, since we already include all relevant and marginal couplings, we expect the decoupling to be robust or at least approximately valid to a good accuracy.

The infrared flow of $Z, D$, and $\gamma$ is determined by the values of the respective anomalous dimensions at the fixed point. Equations (SI-28) and (SI-32) imply the scaling behavior

$$
Z \sim \Lambda^{-\eta_{Z}}, \quad D \sim \Lambda^{-\eta_{D}}, \quad \gamma \sim \Lambda^{-\eta_{\gamma}}
$$

for $\Lambda \rightarrow 0$. While $\eta_{D}$ and $\eta_{\gamma}$ describe the flow of real quantities and are, therefore, themselves real by definition, $\eta_{Z}$ is in general a complex valued function of $\mathbf{r}$ and $\mathbf{s}$. At the fixed point, however, the imaginary part vanishes,

$$
\operatorname{Im} \eta_{Z}=0 \text {, }
$$

which ensures scale invariance of the full effective action at the critical point.

As is indicated in the main text, the emergence of $O(2)$ model critical properties in the sector $\mathbf{s}$ is due to the scaling relation $\eta_{Z}=\eta_{\bar{\gamma}}$, which ensures that these anomalous dimensions compensate each other in the $\beta$-functions for the couplings s. (The anomalous dimensions $\eta_{\bar{\gamma}}$ and $\eta_{\gamma}$ associated with the bare and renormalized noise vertices, respectively, are related via $\eta_{\gamma}=\eta_{\bar{\gamma}}-2 \operatorname{Re} \eta_{Z}$, as follows from Eq. (4) in the main text.) This can be seen most simply by expressing, e.g., $\tilde{\kappa}$ in terms of bare quantities,

$$
\tilde{\kappa}=\frac{\gamma \operatorname{Im}(u)}{2 \Lambda \operatorname{Im}(K)^{2}}=\frac{\gamma \operatorname{Im}(\bar{u} / Z)}{2 \Lambda \operatorname{Im}(\bar{K} / Z)^{2}} .
$$

In this form it is apparent that the scaling $\sim \Lambda^{-\eta_{Z}}$ which applies to both $Z$ and $1 / \gamma$ drops out. Similar arguments hold for $w$ and $\tilde{\kappa}^{\prime}$. Alternatively, the cancellation of $\eta_{Z}$ and $\eta_{\gamma}$ in the $\beta$-functions can be seen explicitly by inserting Eqs. (SI-29) and (SI-30) in (SI-33). What remains is a dependence on $\eta \equiv \eta_{D}+\eta_{Z}$ which is just the anomalous dimension associated with the bare kinetic coefficient $\bar{K}$.

\section{GINZBURG CRITERION}

We estimate the extent of the universal critical domain governed by the linearized regime of the Wilson-Fisher fixed point, which provides us with an estimate of both the extent of 
the thermalized regime as well as the energy resolution necessary to probe the critical behavior. This is done by calculating the Ginzburg scale, i.e., the distance from the phase transition where fluctuations on top of the quadratic Bogoliubov-type theory become dominant [11]: We equate the bare distance from the phase transition $\chi$ to the corresponding one-loop correction, yielding

$$
\chi_{G}=\frac{1}{D^{3}}\left(\frac{\gamma \kappa}{4 \pi}\right)^{2}
$$

Here, the parameters $\gamma, \kappa$, and $D$ are those appearing in the mesoscopic description of the system. In the case of excitonpolariton condensates, $\gamma$ and $\kappa$ can thus be read off from the dGPE and the noise correlator [14]. The parameter $D$ typically does not appear explicitly in this description. However, it is included effectively in a complex prefactor of the time derivative in the dGPE ( $m_{L P}$ is the mass of the lower polariton)

$$
i(1+i \Delta Z) \partial_{t} \psi=\left(-\frac{1}{2 m_{L P}} \nabla^{2}+\cdots\right) \psi
$$

Such a term results from two physical mechanisms. First, it describes the leading frequency dependence of the pumping process [15]. To account for this effect, a convenient parameterization is $\Delta Z=P /\left(2 \Omega_{K}\right)$ which is proportional to the pumping strength $P$, and where $\Omega_{K}$ is the gain cutoff frequency. Second, it results from energy relaxation due to scattering of the lower polaritons with high frequency photons and excitons [16]. These processes are captured by the form $\Delta Z=\kappa \bar{n} / 2$ scaling linearly with the time averaged density $\bar{n}$, and a phenomenological relaxation constant $\kappa$.

Dividing the dGPE (SI-41) by $1+i \Delta Z$ leads to an effective kinetic term $-\frac{1-i \Delta Z}{1+\Delta Z^{2}} \frac{\nabla^{2}}{2 m_{L P}} \psi$, resulting in a mesoscopic coherent propagation coefficient $A=\frac{1}{1+\Delta Z^{2}} \frac{1}{2 m_{L P}}$ and an effective mesoscopic diffusion constant $D=\frac{\Delta Z}{1+\Delta Z^{2}} \frac{1}{2 m_{L P}}$ entering Eq. (1) in the main text, and the above Ginzburg criterion.

Finally, we would like to contrast the Ginzburg scale to a scale identified in $[14,15]$. This scale indicates a crossover between a sonic and a purely diffusive excitation spectrum within the symmetry broken phase, and takes the value $\omega_{c}=$ $\kappa \rho_{0}$.

The Ginzburg scale identifies the frequency scale below which critical fluctuations become more dominant than the "bare" terms, which occur in Bogoliubov theory. It is therefore only meaningful - and makes a statement about - the physics close to the phase transition, where the order parameter goes to zero.
Instead, the crossover scale in $[14,15]$ is determined within the symmetry broken phase and is obtained within Bogoliubov theory, without the need of a calculation of fluctuation corrections. This is justified, because within the symmetry broken phase there are no critical fluctuations and mean field plus Bogoliubov theory are valid.

As implied by this comparison, these two scales are not directly related to each other and address different physical questions. In particular, the crossover scale in $[14,15]$ tends to zero when approaching the phase transition by construction, $\rho_{0} \rightarrow 0$. Therefore, in the vicinity of the critical point, the discussion of diffusive vs. coherent dynamics is a more subtle issue. How it works quantitatively is addressed by the calculation of the critical exponents, with the key finding of universal decoherence: The coherent dynamics fades out faster than the dissipative one, measured by the critical exponent $\eta_{r}$.

[1] A. Kamenev and A. Levchenko, Advances in Physics, 58(3), 197 (2009).

[2] A. Altland and B. Simons, Condensed Matter Field Theory (Cambridge University Press, 2010).

[3] C. Wetterich, Phys. Lett. B 301, 90 (1993); Z. Phys. C 57, 451 (1993).

[4] J. Berges, N. Tetradis, C. Wetterich, Phys. Rept. 363223 (2002).

[5] M. Salmhofer and C. Honerkamp, Prog. Theor. Phys. 105, 1 (2001).

[6] J. M. Pawlowski, Annals Phys. 322, 2831 (2007).

[7] B. Delamotte, cond-mat/0702365 (2007).

[8] O. J. Rosten, Physics Reports 511, 177 (2012).

[9] I. Boettcher, J. M. Pawlowski, S. Diehl, Nucl. Phys. Proc. Suppl. 228, 63 (2012).

[10] This representation holds for the stationary states that obey $\frac{\delta \Gamma_{\Lambda}}{\delta \phi_{c}}=\frac{\delta \Gamma_{\Lambda}}{\delta \phi_{q}}=0$.

[11] D. J. Amit and V. Martin-Mayor, Field Theory, The Renormalization Group, And Critical Phenomena (World Scientific, Singapore, 2005).

[12] D. Litim, Phys. Lett. B 486, 92 (2000).

[13] J. Berges, G. Hoffmeister, Nucl. Phys. B 813383 (2009).

[14] I. Carusotto and C. Ciuti, Physical Review B 72, 125335 (2005); M. Wouters and I. Carusotto, Phys. Rev. Lett. 99, 140402 (2007); J. Keeling and N.G. Berloff, Phys. Rev. Lett 100, 250401 (2008).

[15] M. Wouters and I. Carusotto, Phys. Rev. Lett. 105, 020602 (2010)

[16] M. Wouters, T. C. H. Liew, and V. Savona, Phys. Rev. B 82, 245315 (2010). 